\title{
O GÊNERO BACCHARIS LINNAEUS, SEÇÃO OBLONGIFOLIAE DC. (ASTERACEAE - ASTEREAE), NO SUL DO BRASIL. ${ }^{1}$
}

\author{
ANABELA SILVEIRA DE OLIVEIRA² JOSÉ NEWTON CARDOSO MARCHIORI ${ }^{3}$
}

\section{RESUMO}

Neste trabalho é realizado um estudo taxonômico do gênero Baccharis L., seção Oblongifoliae DC., no sul do Brasil. Três espécies e uma variedade foram reconhecidas: Baccharis brachylaenoides DC. var. brachylaenoides, B. brachylaenoides DC. var. rufidula (Sch.-Bip. ex Baker) An. S. de Oliveira \& Deble, $B$. nebulae Malag. \& Hatschbach ex An. S. de Oliveira e B. vismioides DC. As espécies são descritas, ilustradas e separadas mediante chave dicotômica.

Palavras-chave: Baccharis, seção Oblongifoliae, Asteraceae, flora sul-brasileira.

\section{ABSTRACT}

A taxonomic study of the genus Baccharis L., section Oblongifoliae DC., is presently realized to southern Brazil. Three species and one variety were recognized: Baccharis brachylaenoides DC. var. brachylaenoides, B. brachylaenoides DC. var. rufidula (Sch.-Bip. ex Baker) An. S. de Oliveira \& Deble, B. nebulae Malag. \& Hatschbach ex An. S. de Oliveira e B. vismioides DC. All species are described, illustrated and segregated in a dichotomous key.

Kew words: Baccharis, section Oblongifoliae, Asteraceae, southern brazilian flora.

\section{INTRODUÇÃO}

O nome Baccharis foi originalmente utilizado por Linnaeus (1753) para designar quatro plantas, das quais apenas $B$. halimifolia possui as características do táxon atual. Esse binômio, aliás, somente no século XX é que foi proposto como espécie-tipo, por Hitchcock \& Green (1930).

De Candolle (1836), em seu famoso "Prodromus", foi o primeiro a estabelecer uma classificação infragenérica para o gênero Baccharis, dividindo-o em oito seções: Trinervatae, Cuneifoliae, Discolores, Oblongifoliae, Caulopterae, Sergilae, Distichae e Lepydophyllae. Para o estabelecimento das mesmas, De Candolle baseou-se sobretudo em características vegetativas; mesmo assim, as cinco primeiras ainda são consideradas válidas pela maioria dos autores atuais (Nesom, 1990, Giuliano, 2001), Sergilae figura na sinonímia da seção Baccharis L., e somente as duas últimas (Distichae e Lepydophyllae) compreendem espécies subordinadas, atualmente, a outros gêneros.

Para a Flora Brasiliensis, Baker (1882) ordenou o gênero em seis seções: Caulopterae, Cuneifoliae, Discolores, Oblongifoliae, Aphyllae e Angustifoliae, as duas últimas correspondendo a proposições novas do autor.

Heering (1902, 1904), o pioneiro em salientar a importância dos caracteres florais para o estabelecimento de grupos infragenéricos em Baccharis, reconheceu cinco subgêneros: Molina (Ruiz \& Pav.) Heering, Stephananthus (Lehm.) Heering, Pteronoides Heering, Tarchonanthoides Heering e Eubaccharis Heering.

1 Artigo recebido em 21/09/2005 e aceito para publicação em 14/10/2005.

2 Bióloga, MSc., bolsista CAPES, doutoranda do Programa de Pós-Graduação em Engenharia Florestal, Centro de Ciências Rurais, Universidade Federal de Santa Maria, CEP 97105-900, Santa Maria (RS). anabela.biol@mail.ufsm.br

3 Engenheiro Florestal, Dr., bolsista de Produtividade em Pesquisa do CNPq, Professor Titular do Departamento de Ciências Florestais, Universidade Federal de Santa Maria, CEP 97105-900, Santa Maria (RS). balduinia@mail.ufsm.br 
A seção Oblongifoliae foi concebida por De Candolle (1836), com base em 59 espécies de características muito heterogêneas. Em 1967, Cuatrecasas revisou a seção, delimitando-a e propondo uma emenda para a mesma.

Barroso (1976), reuniu as espécies correspondentes à seção Oblongifoliae no "grupo Brachylaenoides", reconhecendo cinco espécies e uma variedade para o Brasil: Baccharis brachylaenoides DC., B. brachylaenoides var. polycephala (Sch.-Bip.) Barroso, Baccharis grandimucronata Malag., B. ligustrina DC., $B$. vernonioides $\mathrm{DC}$. e $B$. vismioides DC.

Em recente trabalho de classificação infragenérica das espécies argentinas, Giuliano (2001) reconheceu duas espécies da seção Oblongifoliae para o referido país: Baccharis brachylaenoides DC. e B. grandimucronata Malag.

\section{DESCRIÇÃO DA SEÇÃO}

Baccharis seção Oblongifoliae DC. emend. Cuatrec.,

A. P. de Candolle, Prodromus 5, p. 416, 1836.
J. Cuatrecasas, Revista Acad. Colomb. Ci. Exact. n. 13, p. 87, 1967.

Lectótipo: Molina oblongifolia Ruiz \& Pav. (= Baccharis oblongifolia (Ruiz \& Pav.) Pers.); designado por J. Cuatrecasas (Revista Acad. Colomb. Ci. Exact. n. 13, p. 87. 1967).

= Seção Paniculatae Heering, subseção VI, Jahrb. Hamburg. Wiss. Anst. Beih. n. 21, p. 23, 1904.

Subarbustos normalmente ramosos, de 0,5$5 \mathrm{~m}$ de altura, glabrescentes ou ferrugíneotomentosos. Folhas alternas, peninérveas ou retinérveas, levemente discolores. Capítulos pedunculados, reunidos em inflorescências corimbiformes, em panículas axilares ou panículas terminais. Invólucros masculino e feminino, campanulados. Receptáculo dos capítulos femininos, com páleas lineares, caducas. Flores, 10-50. Corola das flores femininas, tubuloso-filiformes, de ápice denteado. Corola das flores masculinas, tubulosas e de ápice dilatado, pentalobado. Pápus 1-seriado. Aquênios subcilíndricos, 5-7-costados.

\section{CHAVE PARA AS ESPÉCIES}

1a. Capitulescência em cimas corimbiformes de ramos curtos, paucicéfalas (8-25 capítulos). Capítulos com 40-45 flores. 2. Baccharis nebulae Malag. \& Hatschbach

1b. Capitulescência em panículas terminais de ramos longos, multicéfalas (50-200 capítulos). Capítulos com 15-30 flores.

2a. Folhas glabras, linear-lanceoladas a lanceoladas ( $4-13 \mathrm{~cm}$ de comprimento por $0,5-3 \mathrm{~cm}$ de largura), acuminadas no ápice.

1. B. brachylaenoides DC.

3a. Folhas lanceoladas, de 5-13 cm de comprimento por 1,2-3 cm de largura

1a. B. brachylaenoides DC. var. brachylaenoides

3b. Folhas linear-lanceoladas, de 3-9 cm de comprimento por 0,5-1 cm de largura, revolutas na margem.

..1b. B. brachylaenoides DC. var. rufidula (Sch.-Bip. ex Baker) An. S. de Oliveira \& Deble

2b. Folhas ferrugíneo-tomentosas, obovais (4-4,5 cm de comprimento por 1,5-2 cm de largura), obtuso-mucronadas no ápice.

3. B. vismioides DC. 


\section{DESCRIÇÃO DAS ESPÉCIES}

1a. Baccharis brachylaenoides DC. var. brachylaenoides

A. P. de Candolle, Prodromus 5, p. 421, 1836.

TIPO: Rio de Janeiro, Corcovado, leg. Lund. 609.

= Baccharis venulosa DC., Prodromus 5, p. 421, 1836;

= Psila brachylaenoides $($ DC. $)$ Aristeguieta, Fl. Venezuela, v. 10, n. 1, p. 316. 1964.

Subarbusto ramoso, de 2-5 m de altura (Figura 1a). Folhas lanceoladas $(5-13 \mathrm{~cm}$ de comprimento por 1,2-3 $\mathrm{cm}$ de largura), alternas (entrenós de 0,3-2 cm), sésseis, glabras, inteiras ou denteadas na metade superior, acuminadas no ápice e longamente atenuadas na base (Figura 1a, 1b). Capítulos pedunculados (3-12 mm), ordenados em panículas terminais longas (Figura 1a). Invólucro feminino campanulado, de 2,5-3,5 $\mathrm{mm}$ de altura por 3-4 $\mathrm{mm}$ de diâmetro (Figura 1c). Brácteas involucrais dispostas em 3 -séries; as externas, ovadas ( $2 \mathrm{~mm}$ de comprimento por $1 \mathrm{~mm}$ de largura), obtusas no ápice e com margens hialinas, fimbriadas; as internas, ovado-oblongas ou ovado-lanceoladas (2,5-3,5 $\mathrm{mm}$ de comprimento por $1 \mathrm{~mm}$ de largura), de ápice obtuso a agudo e margens hialinas, fimbriadas (Figura 1c). Receptáculo paleáceo; páleas lineares, caducas, acuminadas, de 4-5 mm de comprimento (Figura 1d). Flores femininas, 20-30, com corola tubuloso-filiforme de 2,6-3 $\mathrm{mm}$ de comprimento e ápice denteado (Figura 1d). Estigma exserto ao tubo da corola, bífido, de 3-3,5 mm de comprimento (Figura 1d). Pápus unisseriado, com cerdas de 3,5-4,5 $\mathrm{mm}$ de comprimento (Figura 1d). Aquênios 5-costados, subcilíndricos, de $1,5 \mathrm{~mm}$ de comprimento (Figura 1f). Invólucro masculino campanulado (2,5-3,5 mm de altura por 3-4 mm de diâmetro). Brácteas involucrais dispostas em 3-séries; as externas, ovadas e obtusas no ápice, de 1,5-2 $\mathrm{mm}$ de comprimento por 1-1,5 mm de largura; as internas, obovado-oblongas e obtusas no ápice, de 2,5-3,5 $\mathrm{mm}$ de comprimento por $1 \mathrm{~mm}$ de largura. Flores masculinas, 20-25, com corola tubulosa de $3 \mathrm{~mm}$ de comprimento e ápice 5secto, revoluto; rudimento do estigma de 3-3,5 $\mathrm{mm}$ de comprimento, com ramos curtos e aderidos entre si (Figura 1e). Pápus com cerdas onduladas, de 2,5-3 mm de comprimento (Figura 1e).

Comentários: Baccharis brachylaenoides assemelha-se a $B$ grandimucronata, diferindo pela forma da inflorescência e folhas.

Distribuição e Habitat: A espécie apresenta ampla distribuição na América do Sul, ocorrendo desde a Venezuela, Colômbia, Peru, Guianas e Argentina, até o sudeste e sul do Brasil.

Material examinado: BRASIL: PARANÁ: Balsa Nova, serra São Luis, arbusto de $1,70 \mathrm{~m}$, capítulos creme, matinha à beira da cuesta devoniana, G. Hatschbach 15.785 , s.d. (MBM). Bocaina do Sul, serra, arbusto $2 \mathrm{~m}$, capítulos alvacentos, J. Cordeiro \& E. Barbosa 2.170, 13.V.2004 (MBM 294.805). Campina Grande do Sul, serra do Capivari Grande, arbusto de capítulos creme, $1 \mathrm{~m}$, orla da matinha nebular, G. Hatschbach, 06.VIII.1961 (MBM 75.458). Guaratuba, Pedra Branca de Araraquara, arbusto de $1,5 \mathrm{~m}$, capítulos creme, mata pluvial, em barrancos na beira do rio, s.leg., 17.X.1964 (MBM 75.457). Lapa, Serrinha, rio São Vicente, arbusto $2 \mathrm{~m}$, flores creme, borda da mata ciliar, G. Hatschbach 2.283, 17.VI.1951 (MBM 71.413, SI); ibidem, fazenda Santa Amélia, flores creme, arbusto de $1 \mathrm{~m}$, crescendo no paredão de arenito das margens do rio Iguaçu, G Hatschbach, 17.VI.1951 (MBM 71.412). Morretes, Marumbi, arbusto $3 \mathrm{~m}$, capítulos alvescentes, encosta de morro, mata pluvial baixa, O. S. Ribas 371 \& O. Brunner, 1.IX.1991 (MBM 146.582); ibidem, Serra do Mar, arvoreta, flores creme, 900 m.s.m., A. C. Cervi 2.780, 11.VIII.1989 (MBM 130.826); ibidem, Marumbi, trilha para o pico, km 60 da ferrovia CuritibaParanaguá, 1.000 m.s.m., arbusto de flores brancas a beges, Floresta Atlântica, F. C. da Silva, 09.VIII.1983 (MBM 116.344). Palmeira, rio dos Papagaios, J. Lindeman \& H. Haas 5.816, 5.VIII.1967 (MBM 136.763). ibidem, rio Lajeado, arbusto $2 \mathrm{~m}$, capítulos creme, mata de galeria, $\mathrm{O}$. S. Ribas et al. 1.885, 5.VII.1997 (MBM 212.964). Ponta Grossa, Parque Estadual de Vila Velha, arbusto de ca. 2 m, I. J. Takeda, 17.VII.2001 (ICN 127.128). Prudentópolis, rio dos Patos, arbusto de $2 \mathrm{~m}$, flor creme, capoeira, G. Hatschbach 41.430, 07.IX.1976 (MBM 57.534). São João do Triunfo, fazenda da Cia. Fiat Lux, J. C. Lindeman \& H. Haas 1.902, s. d. (MBM 10.247). São José dos Pinhais, serra do Emboque, arbusto com capítulos creme, matinha nebular, 1.000-1.100 m.s.m., G. Hatschbach 19.641, 29.VIII.1968 (MBM 7.984); ibidem, Zinco, arbusto de 3 m, capítulos creme, da capoeira, G. Hatschbach, 21.VII.1982 (MBM 76.725). Tijucas do Sul, represa de Vossoroca, arbusto de capítulos alvescentes, clareiras da mata, R. Kummrow 950, XII.1976 (MBM 47.939). SANTA 


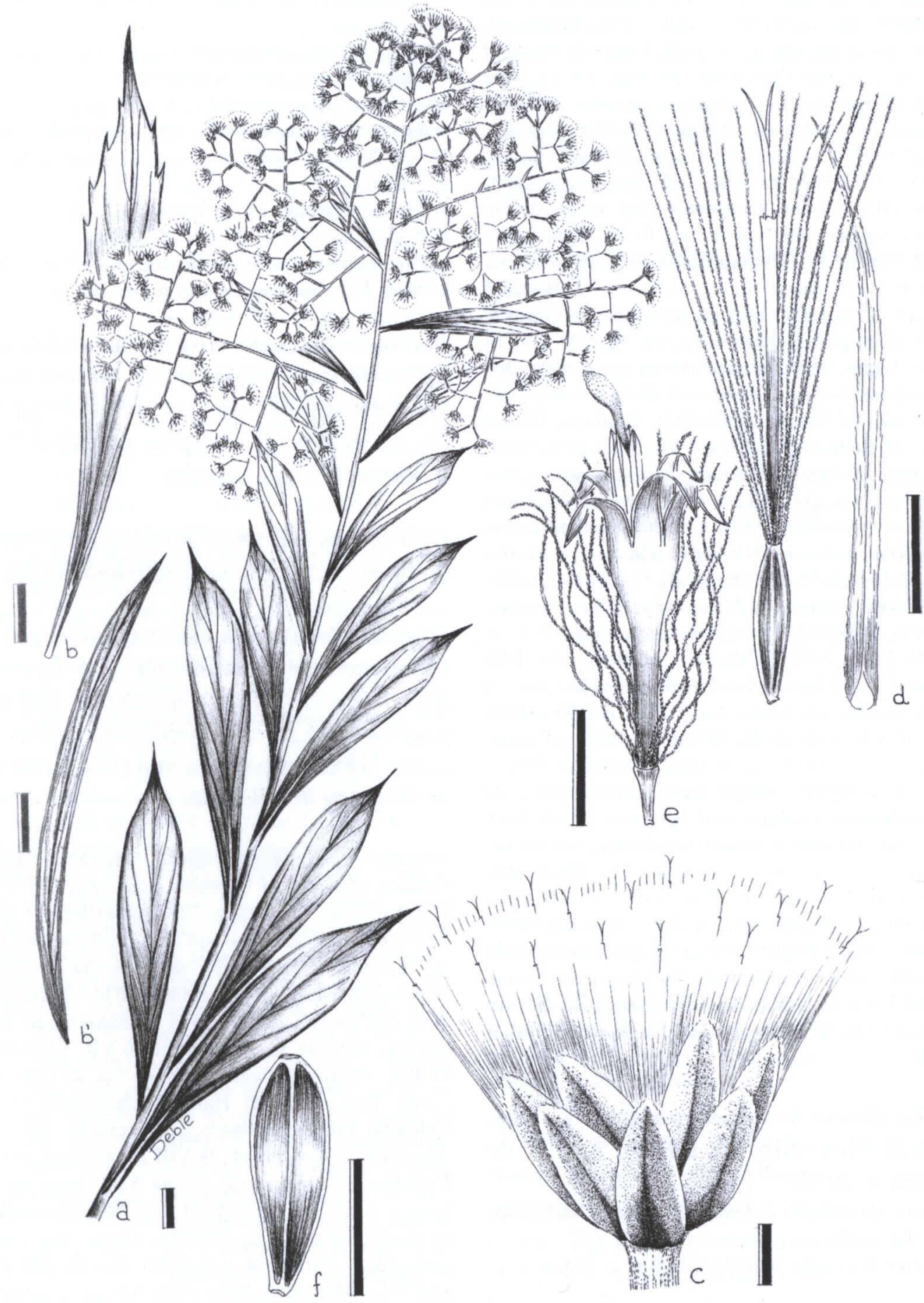

FIGURA 1 - Ramo de exemplar feminino de Baccharis brachylaenoides DC. var. brachylaenoides (a). Folha de outro exemplar (b). Capítulo feminino (c). Flor feminina e pálea do receptáculo (d). Flor masculina (e). Aquênio (f). Folha de B. brachylaenoides var. rufidula (b'). (a, c, d, f, R. Reitz \& Klein 3.394; b, N. Silveira 10.462; b' A. Krapovickas \& C. L. Cristóbal 39.670; e, M. Sobral \& Sevegnani 8.636). 
CATARINA: Biguaçu, serra do Faxinal, in araucarieto, B. Rambo 50.380, 20.VII.1951 (HBR 11.888). Blumenau, morro do Spitzkopf, topo do morro, R. Reitz \& R. M. Klein 8.999, 21.VIII.1959 (PACA 65.289, PEL 3.171). Bom Jardim da Serra, serra do Oratório, capoeira, arbusto 2 m, flor branca, 1.400 m.s.m., R. Reitz \& R. M. Klein 8.662, 19.III.1959 (PEL 3.170); ibidem, serra do Rio do Rastro, arbusto, inflorescência branca, N. Silveira 10.462, 5.V.1991 (HAS 79.449). Chapecó, Pinhal, arbusto de $2 \mathrm{~m}$ de altura, flores creme, 450 m.s.m., R. M. Klein 5.596, 28.VIII.1964 (LP). Florianópolis, in summo monte Cambirela, in rupestribus dumetosis, B. Rambo, 18.VII.1951 (PACA 50.331). Itajaí, morro da Fazenda, mata, R. Reitz \& M. R. Klein 1.905, 01.VII.1954, (PACA 64.950). Lages, Encruzilhada, alto da serra, mata, 900 m.s.m, arbusto de $2 \mathrm{~m}$, flor branca, R. Reitz \& R. M. Klein 12.569, 19.IV.1962 (HBR 41.037). Palhoça, Pilões, capoeira, R. Reitz \& R. M. Klein 3.394, 9.VII.1956 (HBR, MBM, PACA); ibidem, Pilões, capoeira, arbusto $2 \mathrm{~m}$, flor verde-amarelada, 200 m.s.m., R. Reitz \& R. M. Klein 3.386, 9.VII.1956 (HBR, PEL). Rancho Queimado, serra da Boa Vista, mata, 1.000 m.s.m., arbusto de $1 \mathrm{~m}$, flor branca, R. Reitz \& M. R. Klein 9.720, 11.VIII.1960 (HBR 52.059). Santo Amaro da Imperatriz, Pilões, capoeira, 200 m.s.m., arbusto de $2 \mathrm{~m}$, flor verde amarelada, R. Reitz \& R. M. Klein 3.386, 9.VII.1956 (HBR 15.195). RIO GRANDE DO SUL: Cambará do Sul, arbusto 3,5 m, no interior de mata, M. Sobral 2.145, 20.VIII.1983 (MBM 91.835, ICN 85.009). Caxias do Sul, vila Oliva, p. Caxias, in araucarieto, sterilis, B. Rambo, 30.V.1954 (PACA 44.609). Farroupilha, Parque dos Pinheiros, arbusto de aproximadamente $3 \mathrm{~m}$ de altura, Z. Soares et al. 26.IX.1978 (HAS 8.751). Gramado, arbustiva in dumeto, 500 m.s.m., A. Sehnem, 27.XII.1949 (PACA 50.447). Jaquirana, interior mata do Gaspar, R. Wasum 643, 19.VIII.2000 (MBM 16.281). Montenegro, in dumetosis secundariis, B. Rambo, 30.V.1954 (PACA 43.373). São Francisco de Paula, RS 235, na orla da mata, 830 m.s.m., R. Wasum 1.109, 15.VII.2001 (MBM 263.187); ibidem, RS 235, em beira da estrada, R. Wasum 11.222, 19.VIII.2001 (PACA 85.845).

\section{1b. Baccharis brachylaenoides DC. var.} rufidula (Sch.-Bip. ex Baker) An. S. de Oliveira \& Deble

Anabela S. de Oliveira \& Leonardo P. Deble, Balduinia, n. 5, p. 4-5, 2005.

Basiônimo: Baccharis rufidula Sch.-Bip. ex Baker

TIPO: Brasil tropical, leg. Burchell, 4871, s. d.

= Baccharis polycephala Sch.-Bip., Linnaea, 30, p.181, 1859 [nom. illeg., non Weddel, 1956];

= Baccharis brachylaenoides var. polycephala (Sch.-Bip.)
G. M. Barroso, Rodriguésia, v. 28, n. 40, p. 71, 1976 [comb. illeg.];

= Baccharis brachylaenoides var. polycephala (Malag.) Govaerts, World Checklist Seed Plants, v. 2, n. 1-2, p. 9 , 1996 [comb. illeg. superfl.];

= Baccharis brachylaenoides var. polycephala Govaerts, World Checklist Seed Plants, v. 3, n. 1, p. 9, 1999 [nom. nov. illeg.];

= Baccharis pseudopolycephala Malag., Mem. Soc. Cien. Nat. La Salle, v. 37, n. 107, p. 134, 1976;

= Pseudobaccharis polycephala (Sch.-Bip.) Malag., Contrib. Inst. Geobiol. Canoas, n. 8, p. 23, 1957.

Baccharis brachylaenoides var. rufidula difere da variedade típica pelas folhas linearlanceoladas (3-9 cm de comprimento por 0,5-1 $\mathrm{cm}$ de largura) de margens revolutas e pela inflorescência mais ampla.

Distribuição \& Habitat: Restrita ao sul e sudeste do Brasil, convive com a variedade típica.

Observação: A variedade rufidula tem sido freqüentemente confundida com Baccharis ligustrina DC., por ter sido colocada erroneamente na sinonímia desta espécie, por Baker (1882), e assim reproduzida na estampa 24 da Flora Brasiliensis.

Material examinado: BRASIL: PARANÁ: Campina Grande, arbusto $1 \mathrm{~m}$, capítulos creme, matinha nebular, 1.300 m.s.m., Ckoczicki 213, 12.VIII.1969 (MBM 12.016); ibidem, caminho ao Cerro Verde, arbusto de 1,5 m, capítulos creme-claro, margens de córrego, G. Hatschbach, I.1969 (MBM 6.880, MBM 6.881); ibidem, Serra do Capivari, arbusto 1,5 m, capítulos creme, orla da matinha nebular, G. Hatschbach, 6.VIII.1961 (MBM 71.409). Castro, saída para Piraí do Sul, arbusto orla de capão, flor creme, G. Hatschbach (MBM 71.408). Colombo, Embrapa, arbusto $2 \mathrm{~m}$, capítulos creme, mata degradada, G. Hatschbach, 28.VIII.1984 (MBM 169.163). Curitiba, Parque Barigüi, árvore $5 \mathrm{~m}$, flores alvas, C. Kozera et al., X.1996 (MBM 249.055). Mandirituba, arredores, arbusto $2 \mathrm{~m}$, capítulos alvescentes, mata de araucária, R. Kummrow et al. 3.053, 23.VIII.1988 (MBM 123.631). Morretes, Serra Marumbi, p. Morretes, in dumetosis subhumidis, G. Hatschbach 364, 1.IX.1946 (PACA 34.405). Piraí, serra das Furnas, planta da orla de capão, em formação de arenito, A. P. Duarte 5.369, G. Hatschbach, 3.VIII.1960 (PEL 4.863). Pitanga, Borboleta, 
arbusto 1,5 m, capítulo alvacento, faxinal, G. Hatschbach et al., 27.VII.2001 (MBM 262.598). Quatro Barras, morro Mãe Catira, arbusto $2 \mathrm{~m}$, capítulos creme, matinha nebular, 1.200 m.s.m., G. Hatschbach, V.1968 (MBM 3.361). São João do Triunfo, fazenda São João, J. Lindeman \& H. Haas 1.883, 2.VII.1966 (MBM 13.948). São José dos Pinhais, borda do campo, arbusto de $3 \mathrm{~m}$ de altura, capítulos creme, interior da matinha à beira de campo ácido, G. Hatschbach 29.798, 11.VII.1972 (MBM 26.639); ibidem, árvore $7 \mathrm{~m}$, da mata, G. Hatschbach, s.d. (MBM 71.427). São Mateus do Sul, BR 476, km 152, em bosque degradado de araucária, subarbusto de $2 \mathrm{~m}$, A. Krapovickas \& C. L. Cristóbal 39.670, 26.I.1985 (SI). Tibagi, fazenda Monte Alegre, Antas, arbusto da mata secundária, zona da araucária, G. Hatschbach e A. Duarte, 4.VIII.1969 (MBM 71.406). Tijucas do Sul, Piraí, arbusto $2 \mathrm{~m}$, capítulos alvescentes, clareiras da mata, margens de córrego, G. Hatschbach 40.192, s. d. (MBM 57.540). SANTA CATARINA: Biguassú, serra do Faxinal, p. Biguassú, B. Rambo, 20.VII.1951 (PACA 50.380). Blumenau, morro do Spitzkopf, topo do morro, arvoreta, R. Reitz \& R. M. Klein 8.997, 21.VIII.1959 (ICN 2.764); ibidem, região de Mata Ombrófila Densa, Mata Atlântica, arbusto 2-2,5 m, em beira de mata, capítulos brancos, flores masculinas, M. Sobral \& Sevegnani 8.636, V.1998 (MBM 232.439). Bom Jardim da Serra, serra do Oratório, Aparados da Serra, 1.500 m.s.m., R. Reitz \& R. M. Klein 7.173, 18.IX.1959 (HBR 50.904); serra do Oratório, 1.400 m.s.m., $1 \mathrm{~m}$ altura, flor branca, R. Reitz \& R. M. Klein 8.442, 19.II.1959 (HBR, LP 905.025); ibidem, p. São Joaquim, Aparados da Serra, arbusto de $1 \mathrm{~m}$, flor branca, 1.400 m.s.m., R. Reitz \& R. M. Klein 8.443, 19.II.1959 (HBR, LP 905.925). Chapecó, Pinhal, arbusto de $2 \mathrm{~m}$, flor creme, R. M. Klein 5.596, 28.VIII.1969 (LP). Campo Alegre, serra Quiriri, arbusto 1,5 m, capítulo creme, campo de altitude, 1.400 m.s.m., O. S. Ribas et al. (MBM 262.596). Santa Cecília, arredores, arbusto de quase 2 m, flores alvas, E. Pereira 8.394 \& G. Pabst 7.660, 15.I.1964 (PEL 7.156). Timbó, represa do rio Cedro, mata, arvoreta, flor branco-amarelada, R. Reitz \& R. M. Klein 3.518, 19.VII.1956 (PACA 64.985, PEL 2.493). Urubici, Campo dos Padres, arbusto de $1,50 \mathrm{~m}$, capítulos alvescentes, capoeira, junto ao córrego, 1.300 m.s.m., G. Hatschbach 1.991, s. d. (MBM 146.583). Vargem Grande, Lauro Müller, capoeira, arbusto 2 m, 350 m.s.m., R. Reitz \& R. M. Klein 6.708, 11.VII.1958 (HBR 23.129). RIO GRANDE DO SUL: Bom Jesus, Passo da Guarda, interior da mata, fazenda do Cilho, 1.000 m.s.m., R. Wasum 1.542, s. d. (MBM 280.578). Caxias do Sul, Criúva, Ilhéus, em beira de mata, 750 m.s.m., R. Wasum et al., 17.VIII.1988 (MBM 126.075); ibidem, em barranco de capoeira, A. Kegler s.d. (MBM 234.361). Farroupilha, Parque dos Pinheiros, arbusto de aproximadamente $3 \mathrm{~m}$ de altura, com frutos castanhos, Z. Soares et. al., 26.IX.1978 (HAS 8.751). São Francisco de Paula, RS 235, em beira de estrada,
830 m.s.m., R. Wasum 657b, VIII. 2001 (MBM 263.106); ibidem, em beira de caminho, R. Wasum 646, 03.IX.2000 (MBM 263.185); ibidem, beira da estrada, R. Wasum, XII.2001 (MBM 265.192); ibidem, estrada para Taquara, em orla da mata, R. Wasum, 22.IX.2002 (MBM 278.075); ibidem, São José, interior de mata, R. Wasum, 22.IX.2002 (MBM 278.076); ibidem, in araucarieto, B. Rambo, 18.XII.1949 (PACA 44.859).

\section{Baccharis nebulae Malag. \& Hatschbach sp. nov.}

Suffrutex ramosus, 0,5-2 m altus; caulibus superne dense foliosis, inferne subnudis, cicatricosis. Folia obovata ad abovato-oblonga, $1,5-8 \mathrm{~cm}$ longa, 0,5-2 cm lata, alterna (internodiis 0,1-0,8 cm), sessilia, glabra, ápice obtusomucronata, basi attenuata, integerrima vel circa apicem utrinque 1-3-dentata. Capitula brevi pedunculata, cymosocorymbosa disposita. Capitula plantarum feminearum involucrum campanulatum, 5-7 $\mathrm{mm}$ altum, 5-7 $\mathrm{mm}$ crassum. Bracteis involucralibus 3-seriatis; externis, lanceolatis, 3-4 mm longis, 1-1,5 mm latis; interioribus, oblanceolatis, $4-7 \mathrm{~mm}$ longis, $1 \mathrm{~mm}$ latis. Receptaculis paleaceis; paleis membranaceis, linearis, acutis, caducis, 5-7 mm longis. Flores feminei, 40-45; corolla 2-2,8 mm longa, apice dentata. Stylo exserto, 4-5 mm longo. Pappus 4-5 mm longus. Achaenia subcylindracea, 5-costata, 22,2 $\mathrm{mm}$ longa. Capitula plantarum mascularum, 4-6 $\mathrm{mm}$ altum, 5-7 mm crassum. Bracteis involucralibus 3-seriatis; externis oblongis, $2,5 \mathrm{~mm}$ longis, 0,8-1 mm latis, acutis vel obtusis; interioribus, oblanceolatis, acutis, $4 \mathrm{~mm}$ longis, 1-1,5 mm latis. Flores mascula circa 45; corolla 4-4,5 mm longa, apice 5-lobata; stylo 6,5 $\mathrm{mm}$ longo. Pappus 3-3,5 mm longus.

TIPO: BRASIL - PARANÁ, Campina Grande do Sul, pico Caratuva, topo do morro, capítulos alvescentes, 1.950 m.s.m., G. Hatschbach 17.311, 05.X.1967 (MBM 6.837, exempl. masc.). Guaratuba, serra de Araçatuba, arbusto 1 $\mathrm{m}$, capítulo creme, campo de altitude, 1.300 m.s.m., J. M. Silva, E. Barbosa \& J. Cordeiro 3.278, 25.II.2005 (MBM 254.814, exempl. fem.).

Subarbusto ramoso, de 0,5-2 m de altura; ramos densamente folhosos no ápice e normalmente desprovidos de folhas na base (Figura 2a). Folhas obovais a obovado-oblongas (1,5-8 $\mathrm{cm}$ de comprimento por $0,5-2 \mathrm{~cm}$ de largura), alternas (entrenós de 0,1-0,8 cm), sésseis, glabras, obtuso-mucronadas no ápice, de margens crenadas, normalmente inteiras, raramente com 1-3-dentes na metade superior (Figura 2a). Capítulos curtamente pedun- 


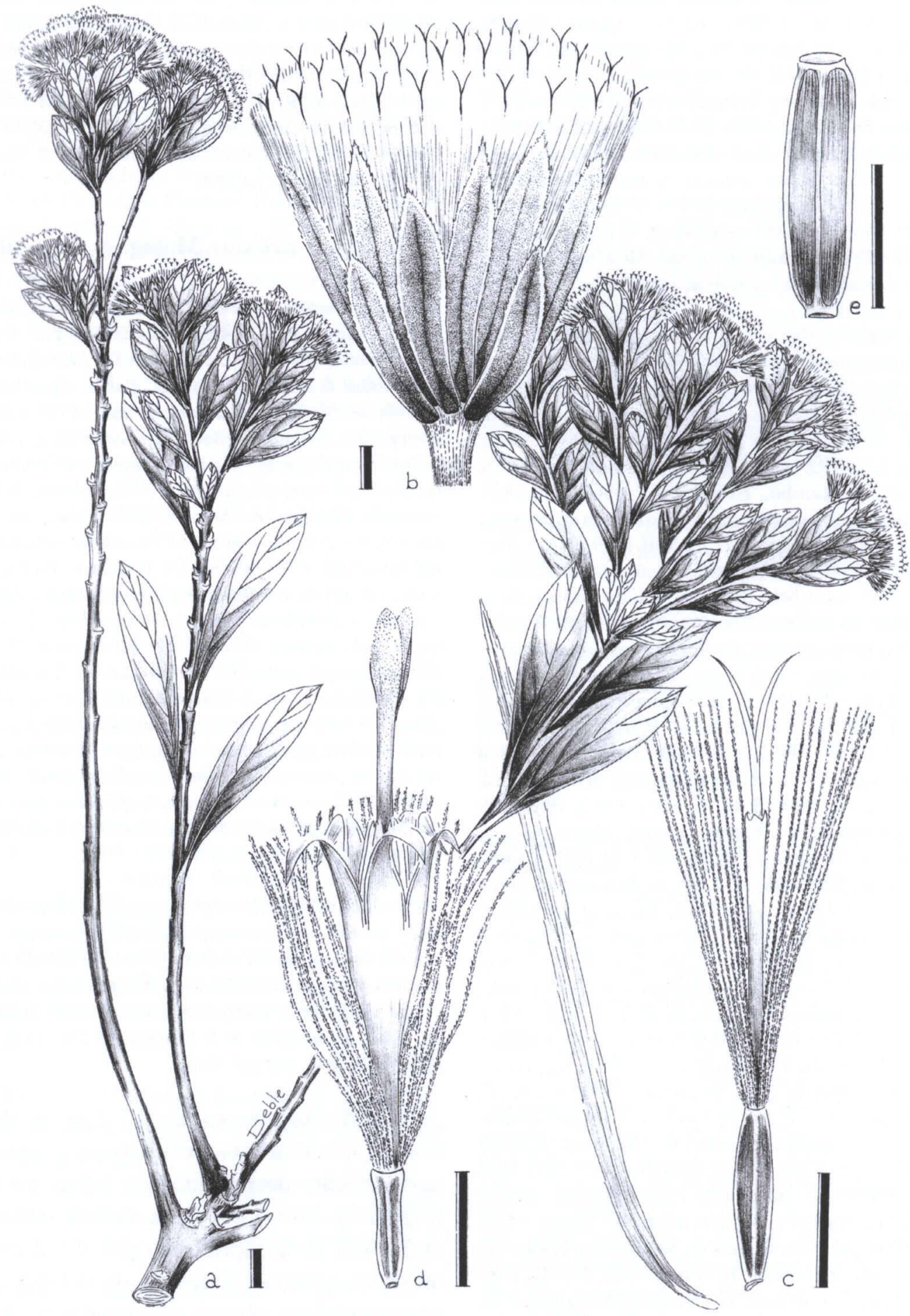

FIGURA 2 - Ramo de exemplar feminino de Baccharis nebulae Malag. \& Hatschbach (a). Capítulo feminino (b). Flor feminina e pálea do receptáculo (c). Flor masculina (d). Aquênio (e). (a, b, c, e, J. M. Silva, E. Barbosa \& J. Cordeiro 3.278; d, G. Hatschbach 17.311). 
culados, ordenados em cimas corimbiformes (Figura 2a). Invólucro feminino campanulado, de 5-7 $\mathrm{mm}$ de altura por 5-7 $\mathrm{mm}$ de diâmetro (Figura 2b). Brácteas involucrais dispostas em 3-séries; as externas, lanceoladas, de 3-4 mm de comprimento por 1-1,5 mm de largura; as internas, oblanceoladas (4-7 $\mathrm{mm}$ de comprimento por $1 \mathrm{~mm}$ de largura), agudas no ápice, com margens hialinas, fimbriadas (Figura 2b). Receptáculo paleáceo; páleas lineares, caducas, agudas, de 5-7 $\mathrm{mm}$ de comprimento. Flores femininas, 40-45, com corola de 2-2,8 $\mathrm{mm}$ de comprimento, com bordo denteado (Figura 2c). Estigma exserto ao tubo da corola, bífido, de 4-5 mm de comprimento (Figura 2c). Pápus unisseriado, com cerdas de 4-5 $\mathrm{mm}$ de comprimento (Figura 2c). Aquênios 5-costados, subcilíndricos, de 2-2,2 $\mathrm{mm}$ de comprimento (Figura 2e). Capítulos masculinos campanulados, com invólucro de 4-6 $\mathrm{mm}$ de altura por 5-7 mm de diâmetro. Brácteas involucrais dispostas em 3-séries; as externas, oblongas (2,5 $\mathrm{mm}$ de comprimento por $0,8-1 \mathrm{~mm}$ de largura), de ápice agudo a obtuso; as internas, oblanceoladas e agudas no ápice, de $4 \mathrm{~mm}$ de comprimento por 1-1,5 mm de largura. Flores masculinas, cerca de 45, com corola de 4-4,5 $\mathrm{mm}$ de comprimento e ápice 5-lobado (Figura $2 \mathrm{~d}$ ); rudimento do estigma de $6,5 \mathrm{~mm}$ de comprimento, com ramos longos e separados entre si (Figura 2d). Pápus com cerdas de 3-3,5 $\mathrm{mm}$ de comprimento (Figura 2d).

Distribuição \& Habitat: A espécie ocorre nos estados do Paraná e Santa Catarina, na orla da matinha nebular e encosta de morros, normalmente em altitude superior a 1.000 m.s.m.

Material examinado: BRASIL: PARANÁ: Campina Grande do Sul, serra Ibitiraquire, trilha para o pico Paraná, arbusto $1,5 \mathrm{~m}$, capítulo creme, matinha nebular, 1.850 m.s.m., J. M. Silva, E. Barbosa \& O. S. Ribas 2.054, 5.X.1997 (MBM 217.708). Guaratuba, serra de Araçatuba, arbusto de $1,70 \mathrm{~m}$, capítulos creme, mata nebular, 1.300 m.s.m., C. B. Poliquesi 201 \& J. Cordeiro, 9.XI.1994 (MBM 170.267); ibidem, subarbusto $50 \mathrm{~cm}$, capítulo creme, matinha nebular, 1.300 m.s.m., J. M. Silva, E. Barbosa \& J. Cordeiro 3.260, 25.II.2000 (MBM 254.055). Piraquara, serra do Emboque, arbusto $1 \mathrm{~m}$, capítulos creme, matinha nebular, 1.200 m.s.m., G. Hatschbach 25.747, 3.XII.1970 (MBM 17.827). Quatro Barras, morro Mãe Catira, arbusto de $1 \mathrm{~m}$, capítulos creme, matinha nebular, 1.300 m.s.m., J. M. Silva 662 \& C.B. Poliquesi, 31.X.1999 (MBM 131.739); ibidem, subarbusto $60 \mathrm{~cm}$, flor creme, feminina, encosta do morro, R. Kummrow 2.631 \& F. J. Zelma, 8.X.1985 (MBM 105.002); ibidem, subarbusto de $1 \mathrm{~m}$, capítulo creme, mata nebular do cume e encosta do morro, R. Kummrow 2.622 \& J. M. Silva, 8.X.1985 (MBM 105.004). SANTA CATARINA: Campo Alegre, subida para a Serra Quiriri, arbusto 1,30 $\mathrm{m}$ de altura, flor creme, campo de altitude, 1.500 m.s.m, J. Cordeiro, J. M. Silva, E. Barbosa \& O. S. Ribas 1.700 , 28.XII.1999 (MBM 243.962); ibidem, arbusto 1,70 m, capítulos alvacentos, matinha nebular, 1.000 m.s.m. J. Cordeiro 938 \& E. Barbosa, 19.XI.1992 (MBM 155.282).

Comentários: A espécie demonstra afinidade com Baccharis grandimucronata Malag., diferindo pelas folhas de bordo inteiro ou com 1-3 dentes, pela inflorescência paucicéfala e pelos invólucros maiores, com número maior de flores por capítulo. De Baccharis meridensis Steyermark, difere pelo menor número de capítulos na inflorescência, pelo ápice mucronado das folhas e pelas folhas basais obovado-oblongas.

Observações: Baccharis nebulae foi reconhecida como nova espécie por Malagarriga \& Hatschbach, que enviaram o tipo para ser publicado por Graziela Maciel Barroso, na época envolvida com a revisão do gênero Baccharis para o Brasil. Dispondo de escasso material, a autora identificou-o como $B$. grandimucronata. Com a recente ampliação no número de coletas, foi possível constatar que o táxon encerra características suficientemente distintas para seu reconhecimento como espécie.

\section{Baccharis vismioides DC.}

A. P. De Candolle, Prodromus 5, p. 412. 1836.

TIPO: São Paulo, Sellow HIB 478. Foto SI! 37746 


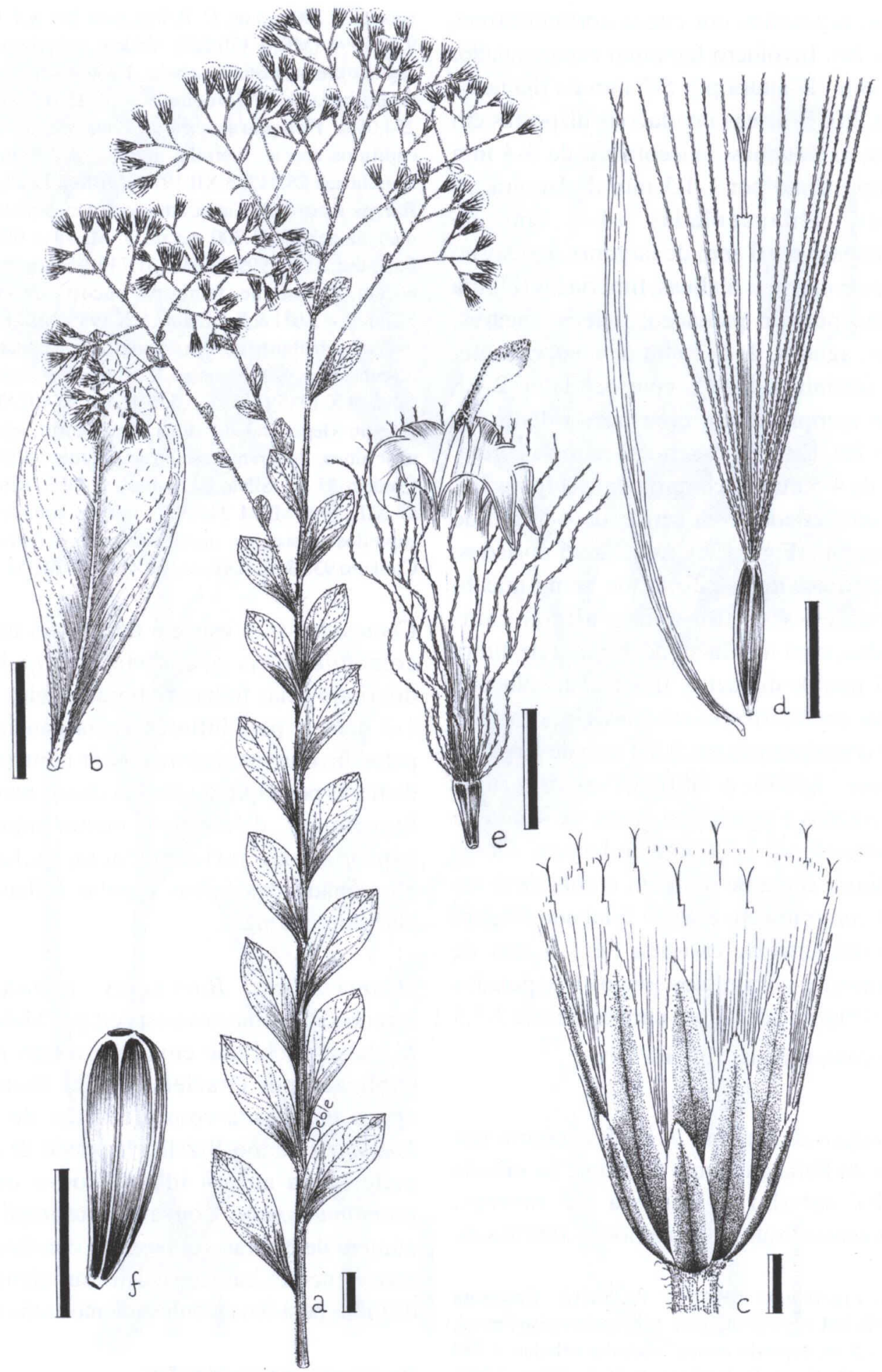

FIGURA 3 - Ramo de exemplar feminino de Baccharis vismioides DC. (a). Folha (b). Capítulo feminino (c). Flor feminina e pálea do receptáculo (d). Flor masculina (e). Aquênio (f). (a, b, c, d, f, G. Hatschbach 5.986; e, G. Hatschbach, 5.985). 
Subarbusto com cerca de $60 \mathrm{~cm}$ de altura, ereto, folhoso até o ápice (Figura 3a). Folhas obovais, (4-4,5 cm de comprimento por 1,5-2 cm de largura), alternas (entrenós de 0,6-1,2 cm), sésseis, inteiras, obtuso-mucronadas no ápice, atenuadas na base, cobertas por pubescência ferrugínea (Figura 3b). Capítulos pedunculados, ordenados em panículas terminais longas (Figura 3a). Invólucro feminino de 3,5-5 mm de altura por $4 \mathrm{~mm}$ de diâmetro (Figura 3c). Brácteas involucrais dispostas em 3-4-séries; as externas, lanceoladas ( $2 \mathrm{~mm}$ de comprimento por $1 \mathrm{~mm}$ de largura); as internas, oblongolanceoladas e levemente agudas no ápice, de 34,5 mm de comprimento por $1 \mathrm{~mm}$ de largura (Figura 3c). Receptáculo paleáceo; páleas lineares, caducas, agudas, de $5 \mathrm{~mm}$ de comprimento (Figura 3d). Flores femininas, 1215 , com corola tubuloso-filiforme $(3 \mathrm{~mm}$ de comprimento), denteada no ápice (Figura 3d). Estigma exserto ao tubo da corola, bífido, de 4 $\mathrm{mm}$ de comprimento (Figura 3d). Pápus unisseriado, com cerdas de $5 \mathrm{~mm}$ de comprimento (Figura 3d). Aquênios subcilíndricos, 5-costados, de $2 \mathrm{~mm}$ de comprimento (Figura 3f). Invólucro masculino de 3-4 mm de altura por 4-5 mm de diâmetro. Brácteas involucrais dispostas em 3-séries; as externas, ovadolanceoladas e agudas no ápice, de 1,5 mm de comprimento por $1 \mathrm{~mm}$ de largura; as internas, lanceoladas e agudas no ápice, de 2,5-4 mm de comprimento por $1 \mathrm{~mm}$ de largura. Flores masculinas, cerca de 25 , com corola tubulosa de $5 \mathrm{~mm}$ de comprimento, e ápice 5-lobado, revoluto; rudimento do estigma de $4-5 \mathrm{~mm}$ de comprimento, com ramos curtos, separados entre si (Figura 3e). Pápus com cerdas de 3-4 mm de comprimento (Figura $3 \mathrm{e}$ ).

Distribuição \& Habitat: Endêmica nos estados do Paraná e São Paulo, Baccharis vismioides ocorre em várzeas e terrenos brejosos.

Material examinado: BRASIL: PARANÁ, Jaguariúna, ad rivulum, P. Dusén 11.732, 1.V.1911 (SI). Tibagi, arredores, 696 m.s.m., subarbusto $60 \mathrm{~cm}$, capítulos creme, do brejo, várzea do rio Tibagi, G. Hatschbach 5.985, 05.VI.1959 (MBM 71.433); ibidem, G. Hatschbach 5.986, 05.VI.1959 (MBM 71.434).

\section{AGRADECIMENTOS}

Os autores agradecem a Gert Hatschbach e Osmar Ribas (MBM), a Zilda Dechamps (HBR), a Maria Salete Marchioretto (PACA), a Mara Rejane Ritter (ICN), a Maria de Lourdes Abruzzi Aragão de Oliveira (HAS), a Leila Macias (PEL), a Solon Jonas Longhi (HDCF), a Norma Deginani (SI) e a Laura Iharlegui (LP), pelo acesso ao material examinado, bem como a Leonardo Paz Deble, pela revisão do manuscrito, pelas ilustrações e pela diagnose latina de Baccharis nebulae.

\section{BIBLIOGRAFIA}

Baker, J. Compositae III. Asteroidae. In: Martius, Flora

Brasiliensis. München, Wien, Leipzig, 1882. v. 6, n. 3, p. 1-132, 1882.

Barroso, G. M. Compositae - Subtribo Baccharidinae Hoffmann. Estudo das espécies ocorrentes no Brasil. Rodriguésia, Rio de Janeiro, v. 28, n. 40, p. 1-273, 1976.

Cuatrecasas, J. Revisión de las espécies colombianas del género Baccharis. Revista Academia Colombiana Ciencias Exatas, n. 13, p. 5-102, 1967.

De Candolle, A. P., Prodr. Syst. Nat. Reg. Veget. Paris, 6, p. 398-429, 1838.

Giuliano, D. Clasificación infragenérica de las especies argentinas de Baccharis (Asteraceae, Astereae). Darwiniana, San Isidro, v. 39, n. 1-2, p.131-154, 2001.

Heering, W. C. Compuestas: Baccharis, In: K. F. Reiche (ed.). Estudios críticos sobre la flora de Chile. Anales Univ. Chile, Santiago, n. 111, p. 153-158, 1902.

Heering, W. C. Die Baccharis-Arten der Hamburger Herbars. Jahrb. Hamburg. Wiss. Anst. Beih., n. 21, p. 1-46, 1904.

Linnaeus, C. Species Plantarum, 2, 1753, p. 860.

Malagarriga Heras, R. de P. Index Baccharidinarum. Inst. Geob. La Salle, Porto Alegre, v. 2, p. 1-155, 1952. Malagarriga Heras, R. de P. Nomenclator Baccharidinarum Omnium. Mem. Soc. Cien. Nat. La Salle, Caracas, v. 37 , n. 107 , p. 129-224, 1976. 